\title{
Identification of genes involved in inbreeding depression of reproduction in Langshan chickens
}

\author{
Qian Xue ${ }^{1,2}$, Guohui $\mathrm{Li}^{1,2}$, Yuxia Cao ${ }^{1}$, Jianmei Yin ${ }^{1,2}$, Yunfen Zhu ${ }^{1}$, Huiyong Zhang ${ }^{1,2}$, Chenghao Zhou ${ }^{1,2}$, \\ Haiyu Shen ${ }^{1}$, Xinhong Dou ${ }^{1}$, Yijun Su${ }^{1}$, Kehua Wang ${ }^{1}$, Jianmin Zou ${ }^{1} *$, and Wei Han ${ }^{1,2, *}$
}

\footnotetext{
* Corresponding Authors:

Jianmin Zou

Tel: +86-0514-8559-9000,

Fax: +82-63-270-4037

E-mail: jqszjm@163.com

Wei Han

Tel: +86-0514-8559-9078,

Fax: +86-0514-8559-9078,

E-mail: hanwei830@163.com
}

${ }^{1}$ Poultry Institute, Chinese Academy of Agricultural Science, Yangzhou 225000 China

2 Science and Technology Innovation Center, Poultry Institute of Jiangsu Province, Yangzhou 225000, China

ORCID

Qian Xue

https://orcid.org/0000-0003-0831-439X Guohui Li

https://orcid.org/0000-0002-2870-673X

Yuxia Cao

https://orcid.org/0000-0001-9979-1436 Jianmei Yin

https://orcid.org/0000-0002-2181-5714 Yunfen Zhu

https://orcid.org/0000-0002-3067-6437

Huiyong Zhang

https://orcid org/0000-0002-3876-1337

Chenghao Zhou

https://orcid.org/0000-0002-4539-1979 Haiyu Shen

https://orcid.org/0000-0002-9908-830X Xinhong Dou

https://orcid.org/0000-0001-9737-9544 Yijun Su

https://orcid.org/0000-0002-1901-8484

Kehua Wang

https://orcid.org/0000-0003-4125-1204 Jianmin Zou

https://orcid.org/0000-0002-6328-2573

Wei Han

https://orcid.org/0000-0001-6038-7911

Submitted Apr 21, 2020; Revised Jul 29, 2020 Accepted Oct 5, 2020
Objective: Inbreeding depression of reproduction is a major concern in the conservation of native chicken genetic resources. Here, based on the successful development of strongly inbred (Sinb) and weakly inbred (Winb) Langshan chickens, we aimed to evaluate inbreeding effects on reproductive traits and identify candidate genes involved in inbreeding depression of reproduction in Langshan chickens.

Methods: A two-sample t-test was performed to estimate the differences in phenotypic values of reproductive traits between Sinb and Winb chicken groups. Three healthy chickens with reproductive trait values around the group mean values were selected from each of the groups. Differences in ovarian and hypothalamus transcriptomes between the two groups of chickens were analyzed by RNA sequencing (RNA-Seq).

Results: The Sinb chicken group showed an obvious inbreeding depression in reproduction, especially for traits of age at the first egg and egg number at 300 days $(p<0.01)$. Furthermore, 68 and 618 differentially expressed genes (DEGs) were obtained in the hypothalamus and ovary between the two chicken groups, respectively. In the hypothalamus, DEGs were mainly enriched in the pathways related to vitamin metabolism, signal transduction and development of the reproductive system, such as the riboflavin metabolism, Wnt signaling pathway, extracellular matrix-receptor interaction and focal adhesion pathways, including stimulated by retinoic acid 6 , serpin family F member 1 , secreted frizzled related protein 2 , Wnt family member 6 , and frizzled class receptor 4 genes. In the ovary, DEGs were significantly enriched in pathways associated with basic metabolism, including amino acid metabolism, oxidative phosphorylation, and glycosaminoglycan degradation. A series of key DEGs involved in folate biosynthesis (gamma-glutamyl hydrolase, guanosine triphosphate cyclohydrolase 1), oocyte meiosis and ovarian function (cytoplasmic polyadenylation element binding protein 1 , structural maintenance of chromosomes $1 \mathrm{~B}$, and speedy/RINGO cell cycle regulator family member A), spermatogenesis and male fertility (prostaglandin D2 synthase $21 \mathrm{kDa}$ ), Mov10 RISC complex RNA helicase like 1, and deuterosome assembly protein 1) were identified, and these may play important roles in inbreeding depression in reproduction.

Conclusion: The results improve our understanding of the regulatory mechanisms underlying inbreeding depression in chicken reproduction and provide a theoretical basis for the conservation of species resources.

Keywords: Conservation; Gallus gallus; Inbreeding Depression; Reproduction; Transcriptome

\section{INTRODUCTION}

Inbreeding - mating between related individuals—often occurs in small, isolated, or captive populations. A negative effect of inbreeding is inbreeding depression, which has become a major concern in evolution, ecology, and conservation biology research [1]. Inbreeding depression refers to a reduction in fitness or fitness-related traits due to inbreeding, such as a reduction in juvenile survival rate and reproductive success. Especially, in small-scale 
populations, inbreeding depression in reproduction has significant implications on the ability of populations to evolve and adapt to environmental changes and, eventually, their long-term viability [2]. Thus, inbreeding depression in reproduction is a concern in the conservation of rare and endangered species.

According to classical population genetic studies, inbreeding depression is caused mainly by the phenotypic expression of multiple recessive deleterious mutations and a decreased contribution of over-dominant loci [3]. However, these two hypotheses do not conclusively explain all cases of inbreeding depression. For example, inbreeding depression was found to change with environmental stress in several studies [4], and this was suggested to be associated with the epigenetic modification of DNA [5] and conditional expression of genes [6]. Overall, the mechanisms of inbreeding depression are complex and, therefore, further research should be conducted on different aspects of inbreeding depression at different levels.

Variations in gene expression are pervasively observed among cells, tissues, individuals, populations, and species [7]. Furthermore, most phenotypic differences are related to the regulation of gene expression even in the absence of genetic variation. Previous studies have shown that inbreeding causes changes in the expression of a large numbers of genes [8]. Several studies on Drosophila melanogaster suggested that some of the transcriptomic alterations in inbred individuals were due to inbreeding depression, whereas some have been reported as protective responses against inbreeding depression $[8,9]$. Despite these studies, a detailed regulatory mechanism of transcriptomic alterations in inbreeding depression is not well understood. To the best of our knowledge, most previous studies on inbreeding depression were performed in plants [5] and some animal models such as Mus musculus [10], Drosophila melanogaster [6,8] and Argopecten irradians [11]. So far, studies on genome-wide changes in gene expression in inbred individuals and the molecular mechanisms of inbreeding depression at the transcriptome level in poultry are limited. RNA sequencing (RNA-Seq) is an effective approach to study genome-wide gene expression, and it has been widely used to identify differentially expressed genes (DEGs) regulating various biological processes [12].

In the present study, we aimed to identify a set of candidate genes responsible for inbreeding depression of reproduction in Langshan chicken, a Chinese native chicken breed. We developed strongly and weakly inbred (Winb) chickens; a reduction in the performance of reproductive traits was observed in the strongly inbred (Sinb) individuals. To explore the regulatory mechanism of inbreeding depression in reproduction at the transcriptome level in chicken, we collected tissues from the gonadal axis, including the hypothalamus and ovary, for RNA-Seq.

\section{MATERIALS AND METHODS}

\section{Ethics statement}

All animal experiments were performed in accordance with the protocol of the Animal Use Committee of the Chinese Ministry of Agriculture and were approved by the Animal Care Committee of Chinese Academy of Agricultural Sciences, Beijing, China, under permit No. SYXK(Jing)2019-0046. All efforts were made to minimize animal suffering.

\section{Development of strongly and weakly inbred Langshan Chickens}

A Chinese native chicken breed, the Langshan chicken, conserved at the National Chicken Genetic Resources (Jiangsu, China), was selected as the study species. The conserved population of Langshan chicken was artificially propagated according to the pedigrees to obtain the $\mathrm{F} 1$ generation. We then selected 160 individuals from the F1 generation with similar reproductive performances (within $\pm 10 \%$ of the group mean value); they belonged to 10 families. Subsequently, restriction-site-associated DNA sequencing (RAD-Seq) was conducted on 43 of the 160 individuals (only one was sequenced to represent all the siblings). Based on the results of RAD-Seq, the molecular inbreeding coefficients $\left(\mathrm{F}_{\mathrm{IS}}\right)$ were calculated (ranging from- 0.003 to 0.283 ). The $\mathrm{F}_{\mathrm{IS}}$ of the full siblings was considered to be the same. Finally, individuals with $\mathrm{F}_{\mathrm{IS}}>0.15$ (58 individuals from six families) were selected and crossed with full-sibling mates to obtain a Sinb F2 generation, and individuals with $\mathrm{F}_{\mathrm{IS}}<0.04$ (12 individuals) were crossed, avoiding close relatives, to obtain a Winb F2 generation. The flowchart of generation of strongly and Winb chickens is presented in Figure 1.

\section{Individual selection and tissue collection}

All the chickens in this study were raised under the same standard conditions in a closed chicken house with automatic ventilation, temperature control and humidity adjustment, and fed ad libitum with commercial complete feed. Phenotypic values for some of the reproductive traits (including age at the first egg, weight of the first egg, body weight at the first egg, and egg number at 300 days) were recorded. The IBM SPSS Statistics 20.0 software (International Business Machines Corporation, New York, USA) was used to analyze the data. A two-sample t-test was performed to estimate the differences between the two chicken groups for each trait. The traits with $\mathrm{p}<0.05$ were considered to be significantly different.

Based on the estimated differences in reproductive traits between the two groups, three healthy chickens with reproductive trait values around the group mean values were selected from each of the groups. These six chickens were immediately humanely euthanized. The hypothalamus and 


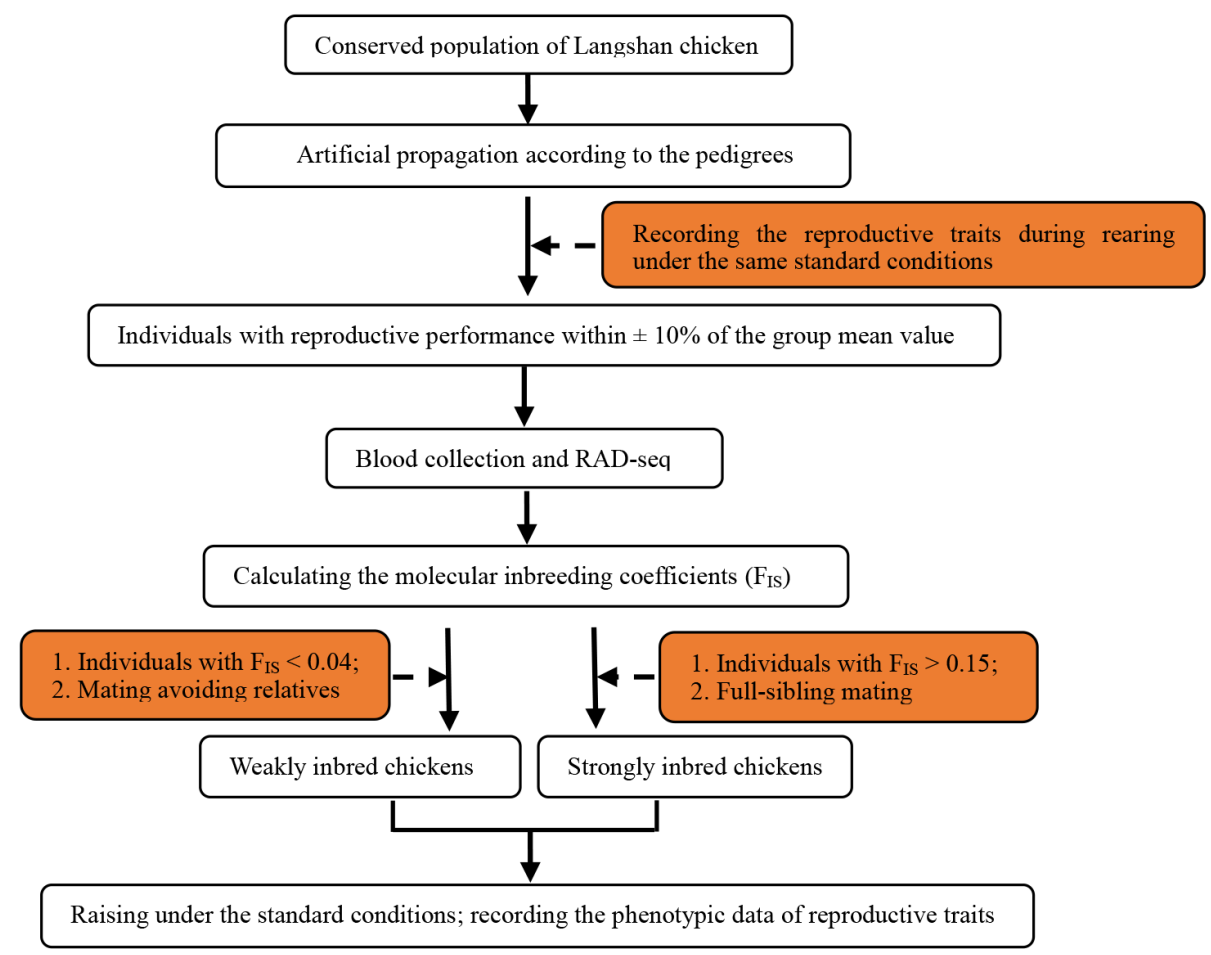

Figure 1. Flowchart of the development of strongly and weakly inbred chickens.

ovary tissues were collected rapidly, snap-frozen in liquid nitrogen and then stored at $-80^{\circ} \mathrm{C}$ until RNA extraction.

\section{RNA-seq library preparation and illumina sequencing} The total RNA from each sample was isolated using TRIzol regent (Invitrogen, Carlsbad, CA, USA). The purity, concentration, and integrity of the RNA were checked using a NanoDrop 2000 spectrophotometer (Thermo Fisher Scientific, Waltham, MA, USA) and an Agilent 2100 Bioanalyzer (Agilent Technologies, Santa Clara, CA, USA), respectively. The RNA integrity number of all samples was greater than 9.5. The polyA mRNA was recovered using oligo(dT) magnetic beads. Thereafter, the RNA was randomly fragmented and transcribed into cDNA. Sequencing adaptors were ligated to the fragments. Single-strand cDNA was then obtained using the Uracil-Specific Excision Reagent (USER) enzyme (NEB, Ipswich, UK). Polymerase chain reaction (PCR) amplification was performed to enrich the cDNA libraries with the following thermal profile: initial incubation at $95^{\circ} \mathrm{C}$ for 2 min, eight cycles of $95^{\circ} \mathrm{C}$ for $30 \mathrm{~s}, 65^{\circ} \mathrm{C}$ for $20 \mathrm{~s}$, and $72^{\circ} \mathrm{C}$ for $45 \mathrm{~s}$; and final incubation at $72^{\circ} \mathrm{C}$ for $7 \mathrm{~min}$. Strandspecific RNA-Seq libraries were then generated. Sequencing was performed using the Illumina Hiseq 2500 instrument to generate 150-bp paired-end reads.

\section{Bioinformatic analysis of RNA-seq data}

The raw data were subjected to quality control using FastQC (http://www.bioinformatics.babraham.ac.uk/projects/fastqc/).
The reads that contained adapters, contaminations, low-quality bases, or undetermined bases were discarded. After passing the quality control step, the reads were mapped to the chicken reference genome (ftp://ftp.ncbi.nlm.nih.gov/genomes/all/ GCF/000/002/315/GCF_000002315.5_GRCg6a/GCF00000 2315.5_GRCg6a_genomic.fna.gz) using HISAT2 [13]. The uniquely mapped reads by alignment were used for annotating transcripts and for determining gene expression levels using the fragments per kilobase per million reads (FPKM) method. The R psych package was used to perform the principle components analysis (PCA) based on the expression levels of all genes. The DESeq2 package was used to calculate differences in gene expression [14]. Genes with a false discovery rate (FDR) of $<0.05$ and fold change of $\geq 2$ were considered to be DEGs between the two groups. The DEGs were then annotated using the gene ontology (GO) and the Kyoto encyclopedia of genes and genomes (KEGG) databases. Functional enrichment analysis was performed using the classic algorithm and Fisher's exact test [15]. The GO terms and KEGG pathways with $\mathrm{p}<0.05$ were considered significantly enriched.

\section{Validation of gene expression by real-time reverse} transcription-quantitative polymerase chain reaction Three DEGs were randomly selected from each of two tissues and were quantified by quantitative polymerase chain reaction (qPCR) to validate the RNA-Seq data. Primer pairs were designed using Primer Premier 5.0 software (PREMIER Bio- 
soft, San Francisco, CA, USA) (Supplementary Table S1). The validation was performed with the same samples used for the RNA-Seq analysis. The total RNA was reverse-transcribed into cDNA using the PrimeScript RT Master Mix kit (TaKaRa Biotechnology Co Ltd, Dalian, China). qPCR was conducted on the Applied Biosystems 7500 real-time PCR system (Applied Biosystems, Foster City, CA, USA), and the reaction mixture with a total volume of $20 \mu \mathrm{L}$ contained 10 $\mu \mathrm{L}$ of SYBR1 Premix Ex Taq $(2 \times), 0.4 \mu \mathrm{L}$ of ROX Reference Dye II (TaKaRa Biotechnology Co, Ltd., China), $0.4 \mu \mathrm{L}$ of each primer $(10 \mu \mathrm{M}), 6.8 \mu \mathrm{L}$ of RNase-free water, and $2 \mu \mathrm{L}$ of CDNA.

The PCR conditions were as follows: initial denaturation at $95^{\circ} \mathrm{C}$ for $30 \mathrm{~s}$, and $40 \mathrm{cycles}$ of denaturation at $95^{\circ} \mathrm{C}$ for $5 \mathrm{~s}$ and annealing at $60^{\circ} \mathrm{C}$ for $34 \mathrm{~s}$. Gene expression levels were normalized to that of $\beta$-actin to obtain relative expression levels using the $2^{-\Delta \Delta C t}$ method [16].

\section{Graphic drawing}

Several types of software were used for generating the figures, such as GraphPad Prism (GraphPad Software Inc., San Diego, CA, USA), Microsoft Office 2013 (Microsoft Corporation, Washington, USA), R package, and the cloud platform of Omicshare (https://www.omicshare.com/tools/).

\section{RESULTS}

Differences in reproductive traits between the Sinb and Winb chickens

As expected, significant differences existed in most of the reproductive traits between the Sinb and Winb chickens, especially for age at the first egg and egg number at 300 days $(p<0.01)$. We also observed apparently reduced reproductive performance in Sinb chickens compared to Winb chickens (Table 1).

\section{Overview of RNA-seq data}

A total of 12 samples from 4 groups were used for RNA-Seq. After quality control, $177.68 \mathrm{~Gb}$ clean data were finally obtained. An average of 54,301,961 and 41,131,453 clean reads were acquired for the ovaries of Winb and Sinb chickens
(Winb_O, Sinb_O), respectively, while 54,775,767 and $47,209,256$ clean reads were acquired for the hypothalamus tissues of the two groups (Winb_H, Sinb_H). The percentage of bases with quality value of $\geq \mathrm{Q} 30$ was no less than $89.26 \%$ for each sample. An average of $88.27 \%$ reads were mapped to the reference genome (Supplementary Table S2). A PCA analysis was performed to confirm the accuracy and reliability of the collected samples. The result showed that the replicates from the same groups clustered relatively closely (Supplementary Figure S1). Thus, the sample collections were reliable, and the sequencing data met the requirements for the subsequent analysis.

\section{Identification of differentially expressed genes}

The expression levels of all genes were calculated as the FPKM using DESeq2. A comparison of gene expression in each of two tissues between the Sinb and Winb chickens was performed. We identified 68 DEGs between Sinb_H and Winb_ H (Sinb_H vs Winb_H), and 618 DEGs between Sinb_O and Winb_O (Sinb_O vs Winb_O) (Supplementary Table S3-S4). Of the 68 DEGs in the hypothalamus, 61 genes were upregulated, and 7 genes were downregulated. Among the 618 DEGs in the ovary, we found 186 genes were upregulated and 432 genes downregulated (Figure 1). Five DEGs were commonly identified in both tissues, including calcium-sensing receptor, prostaglandin D2 synthase $21 \mathrm{kDa}$ (PTGDS), ENSGALG000 00039991, glycosyltransferase 1 domain containing 1 , and dynein, axonemal, heavy chain 12 (Figure 2).

DEGs with extremely significant differences in the comparisons are interesting as they may play important roles in related biological processes. As a result, the top five most differentially expressed genes in each of the two tissues ranked by FDR values were analyzed and obtained, for example, stimulated by retinoic acid 6 (STRA6), nephroblastoma overexpressed and deuterosome assembly protein 1 (DEUP1) genes in the hypothalamus and Mov10 RISC complex RNA helicase like 1 (MOV10L1), ribosomal protein S29, and ENSGALG00000002431 genes in the ovary (Table 2).

Validation of differentially expressed genes

To validate the accuracy of DEGs detected by RNA-Seq, we

Table 1. Differences in reproductive traits between the two chicken groups ${ }^{1)}$

\begin{tabular}{lccc}
\hline \multirow{2}{*}{ Traits } & \multicolumn{2}{c}{ Groups (number of individuals) } & \\
\cline { 2 - 3 } & Sinb (73) & Pinb (44) & Walue \\
\hline Age at the first egg (d) & $173 \pm 15$ & $150 \pm 8$ & 0.001 \\
Egg number at 300 days & $84 \pm 28$ & $98 \pm 15$ & 0.01 \\
Weight of the first egg (g) & $36.1 \pm 3.4$ & $37.1 \pm 3.5$ & 0.814 \\
Body weight at the first egg (g) & $1,753 \pm 149$ & $1,708 \pm 156$ & 0.036 \\
\hline
\end{tabular}

\footnotetext{
1) Phenotypic values of each trait were represented as means \pm standard errors.

2) Sinb is strongly inbred, Winb is weakly inbred

${ }^{3)}$ Comparisons between the two chicken groups based on a two-sample t-test. The traits with $p<0.05$ were considered to be significantly different.
} 

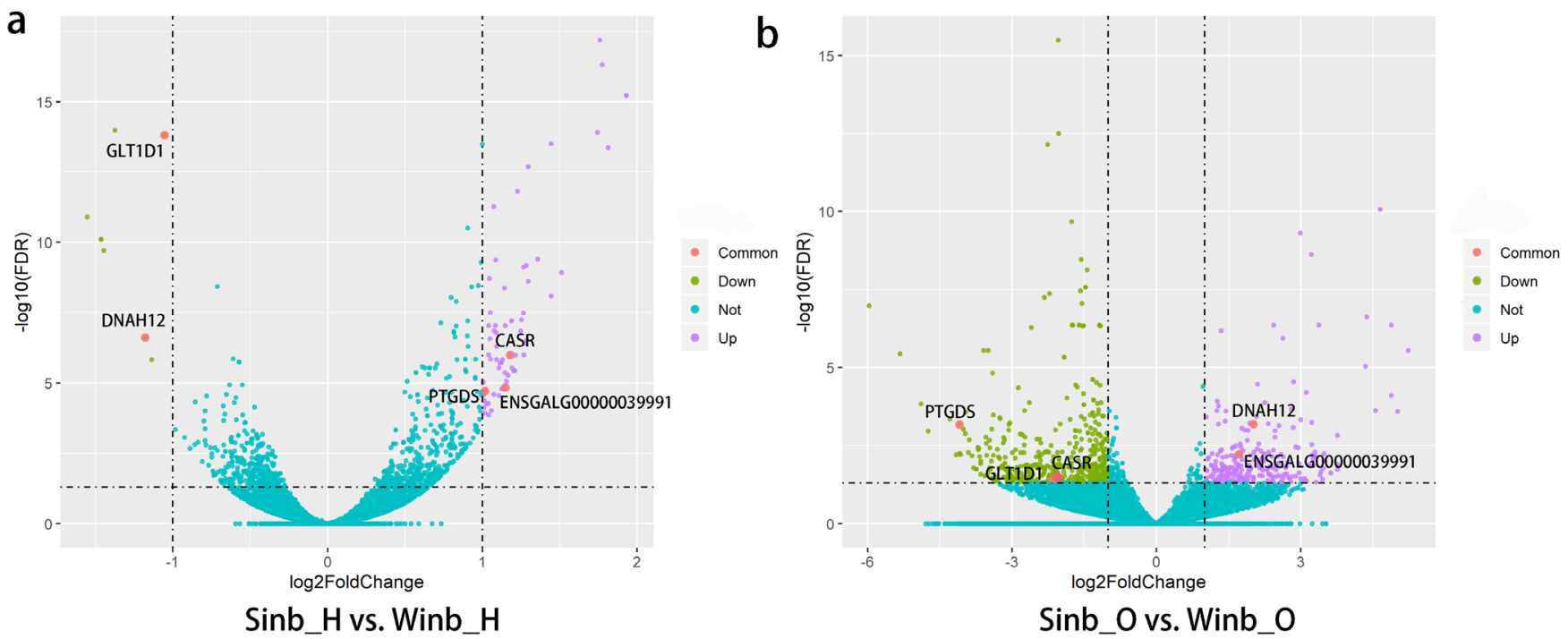

Figure 2. Volcanic map of the analysis of differentially expressed genes (DEGs). Blue dots indicate genes with no significant differences. Purple, green, and pink dots indicate upregulated, downregulated and the common DEGs in each comparison, respectively.

Table 2. Top five most differentially expressed genes ranked by false discovery rate values in each tissue

\begin{tabular}{lllcc}
\hline Tissues & Top 10 DEGs & Description & log2(fold change) & FDR \\
\hline Hypothalamus & STRA6 & Stimulated by retinoic acid 6 & 1.76 & $6.60 \mathrm{E}-18$ \\
& NOV & Nephroblastoma overexpressed & 1.77 & $4.79 \mathrm{E}-17$ \\
& ENSGALG00000041885 & - & 1.93 & $5.98 \mathrm{E}-16$ \\
& ENSGALG00000040576 & - & 1.93 & $5.98 \mathrm{E}-16$ \\
& DEUP1 & Deuterosome assembly protein 1 & -1.37 & $1.04 \mathrm{E}-14$ \\
Ovary & ENSGALG00000002431 & - & -2.04 & $3.28 \mathrm{E}-16$ \\
& RPS29 & Ribosomal protein S29 & -2.03 & $3.13 \mathrm{E}-13$ \\
& ENSGALG00000030318 & - & 4.65 & $8.47 \mathrm{E}-11$ \\
& ENSGALG00000030044 & - & 4.65 & $8.47 \mathrm{E}-11$ \\
& MOV10L1 & Mov10 RISC complex RNA helicase like 1 & 2.99 & $4.87 \mathrm{E}-10$ \\
\hline
\end{tabular}

DEGs, differentially expressed genes; FDR, false discovery rate.

used real-time reverse transcription-qPCR (RT-qPCR) to evaluate the expression levels of six DEGs (apolipoprotein C3, potassium calcium-activated channel subfamily $\mathrm{U}$ member 1 , glycine amidinotransferase from the ovary and macrophage mannose receptor 1-like, carbonic anhydrase $3 \mathrm{~B}$, superoxide dismutase 3 from the hypothalamus). It was found that the RT-qPCR results were consistent with the RNA-Seq results regarding the direction of changes in the expression level of DEGs (Figure 3), which confirmed the validity of the data from RNA-Seq.

\section{Functional analysis of differentially expressed genes} The DEGs identified in the hypothalamus and ovary tissues between the Sinb and Winb chickens may be important for the regulation of inbreeding depression in chicken reproduction. Thus, to further explore the biological function of these genes, functional annotation and enrichment analysis were performed using the GO and KEGG databases. In the hypothalamus, five GO terms were significantly enriched ( $\mathrm{p}<$ 0.05 ) by the DEGs, which were mainly related to the extracellular matrix (ECM) and extracellular region (Supplementary Figure S2). KEGG enrichment analysis of the DEGs revealed that 6 pathways were significantly enriched $(\mathrm{p}<0.05)$ (Figure 4a; Supplementary Table S5), including the Wnt signaling pathway, riboflavin metabolism, ECM-receptor interaction, focal adhesion, advanced glycation end-products and receptor for advanced glycation end-products signaling pathway in diabetic complications and melanogenesis. A total of eight DEGs were involved in these pathways, including serpin family F member 1 (SERPINF1), secreted frizzled related protein 2 (SFRP2), Wnt family member 6 (WNT6), frizzled class receptor 4 (FZD4), ectonucleotide pyrophosphatase/ phosphodiesterase 3 (ENPP3), collagen type VI alpha 1 chain, collagen type I alpha 2 chain, and vascular endothelial growth factor D (Figure 5a).

In the ovary tissues, the DEGs were significantly enriched 


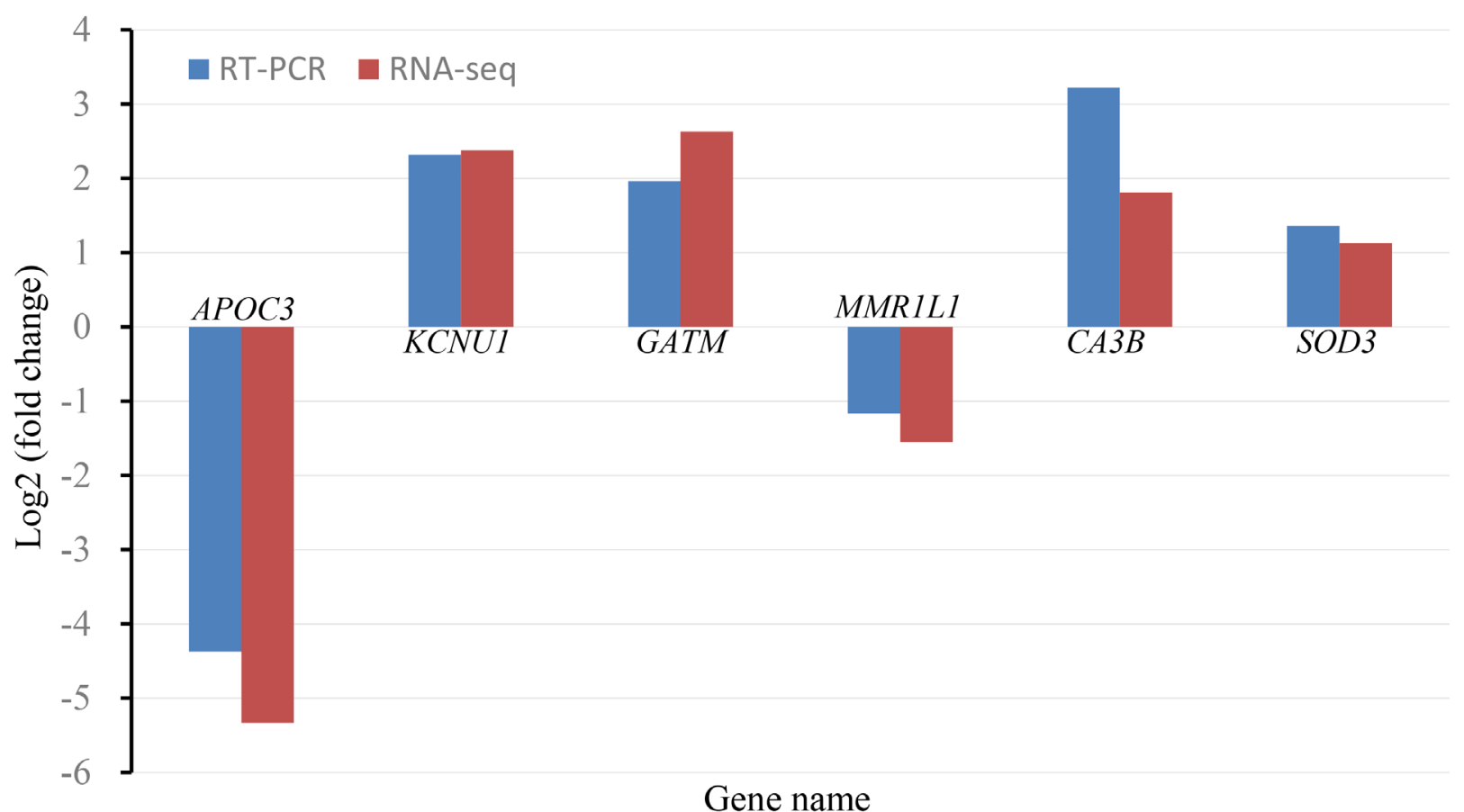

Figure 3. Quantitative polymerase chain reaction (qPCR) validation of differentially expressed genes (DEGs). Changes of expression levels for each gene between Sinb and Winb groups were calculated as log2(fold change). Real-time reverse transcription (RT)-qPCR results were consistent with the RNA-Seq results regarding the direction of changes in the expression level of DEGs.

in GO terms mainly related to ribosomes and components of membranes, and a total of 29 GO terms were significantly enriched $(\mathrm{p}<0.05)$ (Supplementary Figure S3). The results of KEGG enrichment analysis showed that DEGs were sig- a

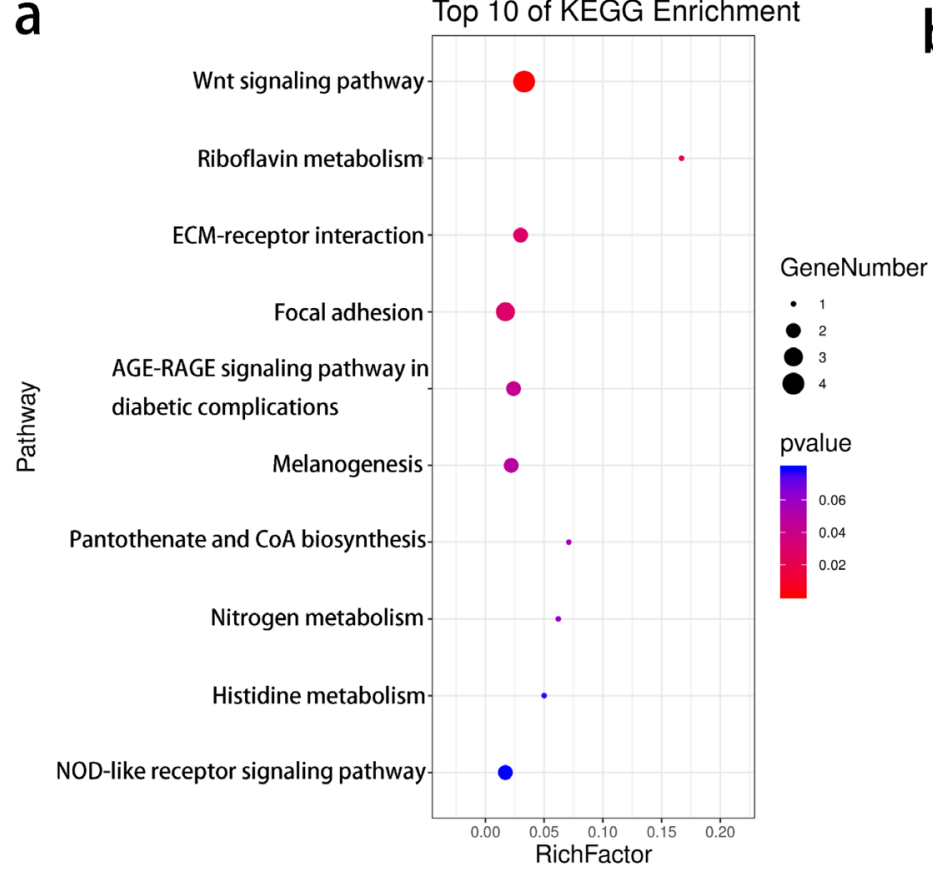

Sinb_H vs. Winb_H b

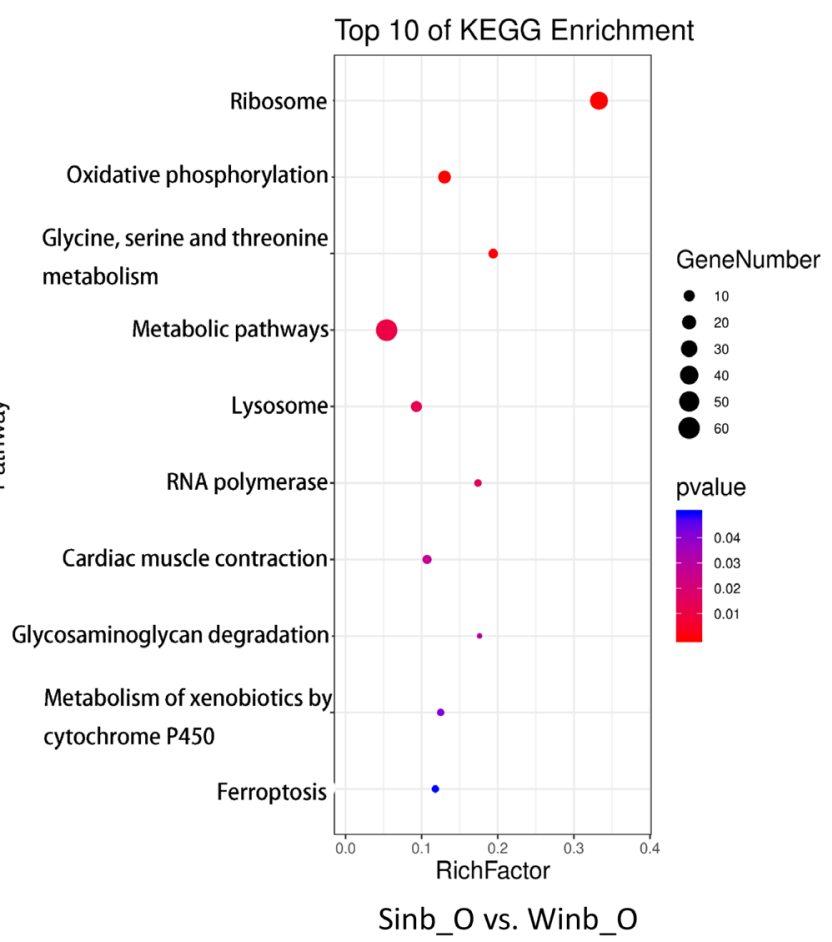

Figure 4. Kyoto encyclopedia of genes and genomes enrichment analysis of differentially expressed genes (DEGs) in the two comparisons. (a) The top 10 most enriched pathways in hypothalamus. (b) The top 10 most enriched pathways in ovary. 
a

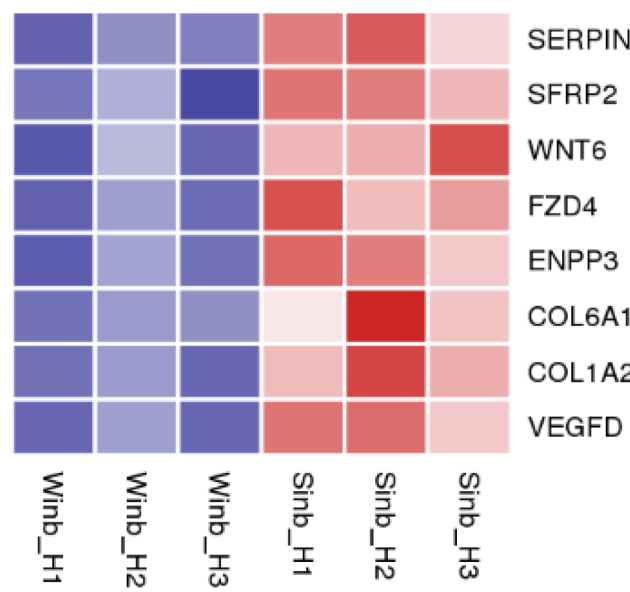

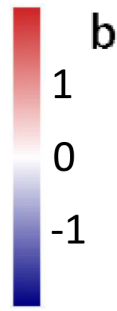

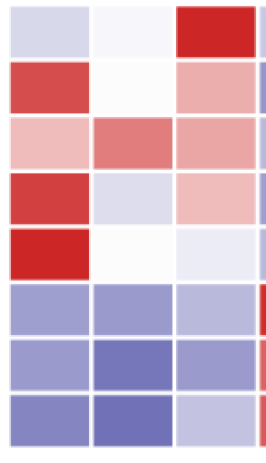

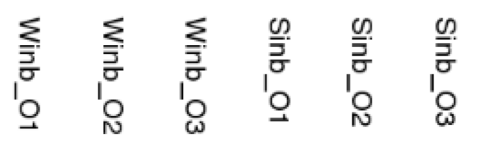

$\mathrm{GCH} 1$

GGH

ENSGALG00000013063

ENSGALG00000028858

LIPA

CPEB1

SMC1B

SPDYA

Figure 5. Heatmap of some of differentially expressed genes (DEGs) in hypothalamus (a) and ovary (b). The colors of bricks from navy to white to red represent the expression levels from low, medium to high.

nificantly enriched in 10 pathways $(\mathrm{p}<0.05)$ (Figure $4 \mathrm{~b}$; Supplementary Table S6). These pathways were mainly related to the process of amino acid metabolism, oxidative phosphorylation, and glycosaminoglycan degradation. In addition, five key pathways related to sex hormone secretion and oocyte development were also enriched, namely, folate biosynthesis, steroid hormone biosynthesis, steroid biosynthesis, oocyte meiosis and progesterone-mediated oocyte maturation, including GTP cyclohydrolase 1 (GCH1), gamma-glutamyl hydrolase (GGH), ENSGALG00000013063, ENSGALG00000028858, lipase A, cytoplasmic polyadenylation element binding protein 1 (CPEB1), structural maintenance of chromosomes 1B (SMC1B), and speedy/RINGO cell cycle regulator family member A (SPDYA) genes (Figure $5 b$ ). These pathways and DEGs may play important roles in the regulation of inbreeding depression in reproduction in Langshan chickens.

\section{DISCUSSION}

In the present study, by comparing the reproductive traits of Sinb and Winb chickens, we found a significant inbreeding depression of reproduction in the Sinb group, especially for the traits of age at the first egg and egg number at 300 days $(\mathrm{p}<0.01)$, which are the most representative in reflecting reproductive performance of female chickens. To explore the molecular mechanism underlying this phenomenon, transcriptomes of tissues from the gonadal axis, such as the hypothalamus and ovary, were compared between Sinb and Winb chicken groups. A total of 68 and 618 DEGs were identified in hypothalamus and ovary, respectively. Of these DEGs, five genes were found to co-exist in both tissues, which inferred that they play important regulatory roles in the gonadal axis. CARS, a calcium-sensing receptor, is central to extra- cellular calcium homeostasis, which is important in many physiological processes such as secretion, neurotransmission, and muscle contraction. A past study showed that CARS played a critical role in early embryogenesis [17]. In our study, CARS was differently expressed in both tissues, indicating it may affect the reproductive performance of Sinb Langshan chickens by regulating neurotransmission and endocrines of the gonad axis. PTGDS, which codes prostaglandin D synthase and catalyzes the conversion of prostaglandin $\mathrm{H} 2$ to prostaglandin $\mathrm{D} 2$, has been reported to be a specific gene involved in male chicken testicular development [18]. As a carrier for lipophilic molecules such as retinoids and thyroid hormones, altering PTGDS levels might influence vitamin A and thyroid hormone availability, and in turn have impacts on diverse biological processes. Thus, the differential expression of these genes in both tissues was inferred to regulate the reproduction of Sinb chicken by complicated biological processes, such as metabolism and secretion.

In addition to PTGDS, MOV10L1, and DEUP1 were also reported to be specifically expressed in testicular tissue $[19,20]$. The expressions of MOV10L1 and DEUP1 between the two chicken groups were extremely different, and they were in the top five ranked by FDR values. MOV10L1 participates in piwi-interacting RNA biogenesis and protects mouse male fertility, enhancing the proliferation capacities of spermatogonial progenitor cells [19]. A study on human tissue-specific expression reported that DEUP1 was specifically highly expressed in testicular tissues, indicating DEUP 1 played an important role in male fertility [20]. Although our study was conducted on female chickens, as we know, a relatively large overlap exists between male and female fertility genes. So, it is speculated that these genes may enhance functions related to male fertility, which affect the reproduction of female chickens. However, the identification of these DEGs 
related to male fertility suggest that the reproduction of male chickens may be more sensitive to inbreeding effect, which points to a new direction for future research.

In the hypothalamus, several DEGs involved in vitamin metabolism were identified, such as STRA6 and ENPP3. STRA6, a cell-surface receptor for the retinol-binding protein, catalyzed vitamin A (retinol) influx, efflux, and exchange, which is involved in diverse biological processes [21]. Based the GO annotation, STRA6 is involved in the biological process of female genitalia development. In our study, STRA6 was found among the top five DEGs, suggesting it may play a critical role in the regulation of inbreeding depression of reproduction in Langshan chickens. Riboflavin, namely vitamin $B_{2}$, has been shown to be essential for good hatchability of eggs. Furthermore, accurate riboflavin supplementation for rats was important for most of the reproductive cycle [22]. KEGG enrichment analysis of DEGs in hypothalamus showed that ENPP3 was significantly enriched in the pathway of riboflavin metabolism, which indicates a probable disorder of riboflavin metabolism in the Sinb chicken groups resulting in depressed reproduction.

Several DEGs in the hypothalamus were enriched in GO terms and KEGG pathways associated with ECM and extracellular region, such as ECM-receptor interaction and focal adhesion. Previous studies have shown that the brain's ECM plays a crucial role in network formation, development, and regeneration of the central nervous system [23]. The interactions of neurons with ECM are important in directing the formation of precise neuronal networks during the development of the nervous system. In addition, focal adhesions have been considered as sites of transmembrane communication between the extracellular environment and the cytoplasm, or links between the ECM and the cytoskeleton. Therefore, it is inferred that high inbreeding in Langshan chickens would affect the formation and development of reproduction-related neuronal networks, leading to changes in signal transduction and neural regulation, and then influencing reproductive performance. Four DEGs (SERPINF1, SFRP2, WNT6, and FZD4) in the hypothalamus were found significantly enriched in the Wnt signaling pathway, which is a complex signaling pathway composed of Wnt, its receptors and regulators to regulate cell differentiation and participate in developmental processes. Studies in mammals have shown that Wnt signaling pathways are also involved in the development of the reproductive system such as the formation of the Mullerian duct and its derivatives, the development of ovarian follicules and mammary glands during pregnancy, ovulation and luteinization, and the establishment of a normal pregnancy [24]. Thus, it was inferred that these four DEGs may regulate the development of the reproductive system of Langshan chickens and affect the reproduction of Sinb chickens.

In the ovary, five key pathways related to sex hormone secretion and oocyte development were enriched, including folate biosynthesis, steroid hormone biosynthesis, steroid biosynthesis, oocyte meiosis and progesterone-mediated oocyte maturation, which may play critical roles in the regulation of inbreeding depression of reproduction in Langshan chickens. Hebert et al [25] studied laying hens and found that egg production was reduced in the absence of folate in the diet. GCH1 and GGH are the key enzymes in the folate pathway $[26,27]$. GCH1 converts guanosine triphosphate into pterin, which is subsequently incorporated into folate derivatives [26]. A previous study showed that GGH regulates the biosynthesis of folic acid in cells and promotes the hydrolysis and outflow of folate and folate antagonists from cells [27]. In our study, these two genes were both downregulated in Sinb chickens, suggesting they may reduce the egg production of Sinb chickens by affecting biosynthesis and derivation of folic acid. CPEB1, SMC1B, and SPDYA were found enriched in progesterone-mediated oocyte maturation and oocyte meiosis pathways, which were significantly highly expressed in the Sinb group. A recent study in a casecontrol analysis showed that significant enrichment of CPEB1 is possibly involved in primary ovarian insufficiency pathogenesis [28]. SMC1B is a meiosis-specific cohesin subunit that is essential for sister chromatid cohesion and DNA recombination. Previous studies have shown that SMC1B maintains the correct meiotic progression in mouse oocytes [29]. SPDYA (Spy1) is a new discovered cell cycle protein capable of promoting cell proliferation dependent on cyclindependent kinase-2 activation. A past study indicated Spyl was probably associated with the proliferation of the epithelial ovarian cancer cells [30]. Therefore, these three genes may cause inbreeding depression of reproduction in Sinb chickens by affecting ovarian function or oocyte meiosis.

In addition, many DEGs identified in the ovary were found enriched in metabolism-related pathways, such as glycine, serine, and threonine metabolism, oxidative phosphorylation and glycosaminoglycan degradation. We speculate that high inbreeding in the population of Langshan chickens affects the basic metabolism, indirectly affects ovarian development, and leads to a decrease in reproductive performance. This finding provides a new direction to further explore the effects of inbreeding on the reproductive performance of poultry.

To our current knowledge, this is the first report on the identification of genes involved in inbreeding depression of chicken reproduction. The results of this study will improve our understanding of the regulatory mechanisms underlying inbreeding depression in chicken reproduction and provide a theoretical basis for the conservation of species resources. More efforts are needed to study the detailed molecular mechanisms of these genes in the regulation of inbreeding depression in chicken reproduction, which will be a challenge in the future. 


\section{CONFLICT OF INTEREST}

We certify that there is no conflict of interest with any financial organization regarding the material discussed in the manuscript.

\section{ACKNOWLEDGMENTS}

This work was supported by the National Natural Science Foundation of China (31572358); Six Talent Peaks Project in Jiangsu Province (NY-024); Project of Key Research and Development Plan of Jiangsu Province (Modern Agriculture) (BE2019353); the Scientific Research Funds of Public Welfare Research Institutes in Jiangsu Province (BM2018026); and the Natural Science Foundation of Yangzhou (YZ2018098). The authors acknowledge all the people who contributed to this study. We would also like to thank Editage (www. editage.cn) for English language editing.

\section{REFERENCES}

1. Reed DH, Fox CW, Enders LS, Kristensen TN. Inbreedingstress interactions: evolutionary and conservation consequences. Ann NY Acad Sci 2012;1256:33-48. https:/doi.org/ 10.1111/j.1749-6632.2012.06548.x

2. Leroy G. Inbreeding depression in livestock species: review and meta-analysis. Anim Genet 2014;45:618-28. https://doi. org/10.1111/age. 12178

3. Roff DA. Inbreeding depression: tests of the overdominance and partial dominance hypotheses. Evolution 2002;56:76875. https://doi.org/10.1111/j.0014-3820.2002.tb01387.x

4. Cheptou PO, Donohue K. Environment-dependent inbreeding depression: its ecological and evolutionary significance. New Phytol 2011;189:395-407. https://doi.org/10.1111/j. 1469-8137.2010.03541.x

5. Vergeer P, Wagemaker N, Ouborg NJ. Evidence for an epigenetic role in inbreeding depression. Biol Lett 2012;8:798801. https://doi.org/10.1098/rsbl.2012.0494

6. Vermeulen CJ, Sørensen P, Gagalova KK, Loeschcke V. Flies who cannot take the heat: genome-wide gene expression analysis of temperature-sensitive lethality in an inbred line of Drosophila melanogaster. J Evol Biol 2014;27:2152-62. https://doi.org/10.1111/jeb.12472

7. Hutter S, Saminadin-Peter SS, Stephan W, Parsch J. Gene expression variation in African and European populations of Drosophila melanogaster. Genome Biol 2008;9:R12. https:// doi.org/10.1186/gb-2008-9-1-r12

8. Garcia C, Avila V, Quesada H, Caballero A. Candidate transcriptomic sources of inbreeding depression in Drosophila melanogaster. PLoS One 2013;8:e70067. https://doi.org/10. 1371/journal.pone.0070067

9. Garcia C, Avila V, Quesada H, Caballero A. Gene-expression changes caused by inbreeding protect against inbreeding depression in Drosophila. Genet 2012;192:161-72. https:// doi.org/10.1534/genetics.112.142687

10.Hoffman JI, Simpson F, David P, et al. High-throughput sequencing reveals inbreeding depression in a natural population. Proc Natl Acad Sci USA 2014;111:3775-80. https:// doi.org/10.1073/pnas.1318945111

11. Wang Y, Zhang S, Zhang G, Cong R, Li L. Molecular mechanism of inbreeding effects based on RNA-Seq analysis of the adductor muscle of bay scallop (Argopecten irradians). Hai Yang Xue Bao 2019;38:44-51. https://doi.org/10.1007/ s13131-019-1351-4

12. Wang X, Wang XW, Wang LK, Feng ZX, Zhang XG. A review on the processing and analysis of next-generation RNA-seq data. Prog Biochem Biophys 2010;37:834-46. https://doi.org/ 10.3724/SP.J.1206.2009.00151

13.Schmieder R, Edwards R. Quality control and preprocessing of metagenomic datasets. Bioinformatics 2011;27:863-4. https://doi.org/10.1093/bioinformatics/btr026

14.Love MI, Huber W, Anders S. Moderated estimation of fold change and dispersion for RNA-seq data with DESeq2. Genome Biol 2014;15:550. https://doi.org/10.1186/s13059014-0550-8

15. Young MD, Wakefield MJ, Smyth GK, Oshlack A. Gene ontology analysis for RNA-seq: accounting for selection bias. Genome Biol 2010;11:R14. https://doi.org/10.1186/gb-201011-2-r14

16.Livak KJ, Schmittgen TD. Analysis of relative gene expression data using real-time quantitative PCR and the $2^{-\Delta \Delta C T}$ method. Methods 2001;25:402-8. https://doi.org/10.1006/meth.2001. 1262

17. Hannan FM, Thakker RV. Calcium-sensing receptor (CaSR) mutations and disorders of calcium, electrolyte and water metabolism. Best Pract Res Clin Endocrinol Metab 2013;27: 359-71. https://doi.org/10.1016/j.beem.2013.04.007

18. Moniot B, Boizet-Bonhoure B, Poulat F. Male specific expression of lipocalin-type prostaglandin $\mathrm{d}$ synthase (cPTGDS) during chicken gonadal differentiation: relationship with cSOX9. Sex Dev 2008;2:96-103. https://doi.org/10.1159/000 129694

19. Fu K, Tian S, Tan H, et al. Biological and RNA regulatory function of MOV10 in mammalian germ cells. BMC Biol 2019;17:39. https://doi.org/10.1186/s12915-019-0659-z

20.Fagerberg L, Hallstrom BM, Oksvold P, et al. Analysis of the human tissue-specific expression by genome-wide integration of transcriptomics and antibody-based proteomics. Mol Cell Proteomics 2014;13:397-406. https://doi.org/10.1074/mcp. M113.035600

21.Kawaguchi R, Zhong M, Kassai M, Ter-Stepanian M, Sun H. STRA6-catalyzed vitamin A influx, efflux, and exchange. J Membr Biol 2012;245:731-45. https://doi.org/10.1007/s00232012-9463-1 
22.Schumacher SMF, Williams MA, Lyman RL. Effect of high intakes of thiamine, riboflavin and pyridoxine on reproduction in rats and vitamin requirements of the offspring. J Nutr 1965;86:343-9. https://doi.org/10.1093/jn/86.4.343

23. Quattromani MJ, Hakon J, Rauch U, Bauer AQ, Wieloch T. Changes in resting-state functional connectivity after stroke in a mouse brain lacking extracellular matrix components. Neurobiol Dis 2018;112:91-105. https://doi.org/10.1016/j. nbd.2018.01.011

24.Zheng P, Vassena R, Latham K. Expression and downregulation of WNT signaling pathway genes in rhesus monkey oocytes and embryos. Mol Reprod Dev 2006;73:667-77. https://doi. org/10.1002/mrd.20428

25. Hebert K, House JD, Guenter W. Effect of dietary folic acid supplementation on egg folate content and the performance and folate status of two strains of laying hens. Poult Sci 2005; 84:1533-8. https://doi.org/10.1093/ps/84.10.1533

26. Lee CS, Salcedo E, Wang Q, Wang P, Sims PFG, Hyde JE. Char- acterization of three genes encoding enzymes of the folate biosynthetic pathway in Plasmodium falciparum. Parasitology 2001;122:1-13. https://doi.org/10.1017/S0031182000006946

27. Kim SE, Cole PD, Cho RC, et al. $\gamma$-Glutamyl hydrolase modulation and folate influence chemosensitivity of cancer cells to 5-fluorouracil and methotrexate. Br J Cancer 2013;109:217588. https://doi.org/10.1038/bjc.2013.579

28. Bestetti I, Castronovo C, Sironi A, et al. High-resolution arrayCGH analysis on 46,XX patients affected by early onset primary ovarian insufficiency discloses new genes involved in ovarian function. Hum Reprod 2019;34:574-83. https:// doi.org/10.1093/humrep/dey389

29. Miao Y, Zhou C, Cui Z, et al. Smc1 $\beta$ is required for activation of SAC during mouse oocyte meiosis. Cell Cycle 2017;16:53644. https://doi.org/10.1080/15384101.2017.1282583

30.Lu S, Liu R, Su M, et al. Spy1 participates in the proliferation and apoptosis of epithelial ovarian cancer. J Mol Histol 2016; 47:47-57. https://doi.org/10.1007/s10735-015-9646-Z 